\title{
Controlling the decoration of the reduced graphene oxide surface with pyrene functionalized gold nanoparticles
}

\author{
Luca Gabrielli, ${ }^{1}$ Giulia Altoè,, ${ }^{1}$ Mareen Glaeske, ${ }^{2}$ Sabrina Juergensen, ${ }^{2}$ Stephanie Reich, ${ }^{2}$ Antonio \\ Setaro, ${ }^{2}$ Enzo Menna, ${ }^{1}$ Fabrizio Mancin ${ }^{1}$ and Teresa Gatti ${ }^{1 *}$ \\ ${ }^{1}$ Department of Chemical Sciences, University of Padova, via Marzolo 1, 35131, Padova, Italy \\ ${ }^{2}$ Physics Department, Free University Berlin, Arnimallee 14, 14195 Berlin, Germany.
}

\begin{abstract}
We exploited a non-covalent approach based on $\pi$-stacking interactions to address the formation of hybrids between pyrene-functionalized gold nanoparticles (PyAuNPs) and reduced graphene oxide (RGO), in which the former are distributed homogeneously on the surface of the latter with a high degree of coverage. We used water soluble PyAuNPs of two different average dimensions, namely 2 and $8 \mathrm{~nm}$, in which the pendant pyrene moieties were introduced within a mixed monolayer with a choline derivative. The combination with RGO originates highly insoluble materials, in which microscopy evidences a complete adhesion of the PyAuNPs onto the carbon nanomaterial layers in a highly homogeneous fashion, with no traces of free particles, confirming the high affinity between pyrene-functionalized species and conjugated carbon nanostructure surfaces.
\end{abstract}

\section{Keywords}

Reduced graphene oxide, gold nanoparticles, functional nanohybrids

\section{Introduction}

The combination of graphene based materials ${ }^{[1]}$ (GBMs) with gold nanoparticles ${ }^{[2]}$ (AuNPs) is extremely promising for the preparation of functional hybrid materials for catalysis, ${ }^{[3,4]}$ photonics, ${ }^{[5]}$ electronics, ${ }^{[6,7]}$ sensing $^{[8-12]}$ and various kind of biomedical ${ }^{[8,13]}$ applications. Both materials have in fact interesting properties per se: GBMs demonstrate outstanding electrical ${ }^{[14]}$ and thermal ${ }^{[15]}$ conductivities coupled to mechanical strength ${ }^{[16]}$ and high optical transparency, ${ }^{[17]}$ whereas AuNPs have tunable absorption properties depending on the size and shape, can induce metal-enhanced fluorescence in fluorophores ${ }^{[18]}$ and can be stabilized with a large plethora of organic moieties exploiting alkanethiols-gold bonds, thus also introducing further selective functions. ${ }^{[19-21]}$ Among GBMs, reduced graphene oxide (RGO) emerges as a good compromise between materials with impressive electronic properties, such as those which can be prepared through chemical vapor deposition of suitable precursors on top of a catalytic surface, ${ }^{[22]}$ and materials that can be produced 
on a large scale with limited costs. ${ }^{[23]}$ It is in fact obtained via a two-step procedure starting from largely available natural graphite. ${ }^{[24]}$

Strategies to produce RGO-AuNPs composites normally involve the in situ reduction of a suitable gold precursor in the presence of the GBM, or of its oxidized form, graphene oxide (GO), which is also reduced to RGO during the process. ${ }^{[7,25-27]}$ Defects and oxygen residues on the surface of RGO can act as nucleating sites for the growth of the metal nanoparticles. This approach can be highly efficient and easy to perform, but also very difficult to control in terms of achieving uniform sizes for the in situ prepared AuNPs as well as in the homogeneity of their distribution onto the RGO substrate. Ex situ assembly of previously prepared AuNPs on RGO can be exploited as an alternative strategy, allowing to start from nanoparticle species of which it is easier to control both the shape and size distribution and which can feature particular functionalities aimed at establishing high affinity interactions with the GBM. Also in this case, the presence of oxygenated functional groups on RGO can furnish a valuable tool to build up covalent bonds with AuNPs, if the proper reactive functional groups are introduced on these last ones. Alternatively carbon-carbon double bonds on RGO can be involved in other types of reactions, such as electrophilic additions or cycloadditions. ${ }^{[28,29]} \mathrm{A}$ milder alternative to the use of covalent chemistry involves the so-called non-covalent functionalization of RGO by means of $\pi$-conjugated molecular systems, mainly pyrene, anthracene and naphthalene, but also more complex ones containing heteroatoms. ${ }^{[30]}$ As stated before, being the chemistry of AuNPs so versatile in terms of chemical functionalities which can be appended to the gold surface through Au-S bonds, making use of selected alkanethiols, one can easily design and synthesize AuNPs featuring pendant $\pi$-conjugated molecules. Brunetti et al. made use of AuNPs capped with an alkanethiol functionalized 9,10-di(1,3-dithiole-2-ylidene)-9,10-dihydroanthracene (exTTF) to prepare non-covalent nanohybrids with exfoliated graphene. ${ }^{[31]}$ They demonstrated that analogous AuNPs lacking the exTTF moiety were not interacting with graphene, thus highlighting the key role played by $\pi-\pi$ stacking interactions in driving the formation of the nanohybrid. Furthermore, the exTTF functionalized AuNPs were shown to be effective in binding to graphene because of the presence of multivalent interactions, whereas by employing simple exTTF molecules no binding was achieved. Notwithstanding that well-defined graphene-AuNPs species were obtained following this approach, no homogeneous dispersion of the particles on the graphene surface was evident from transmission electron microscopy (TEM) images, but rather islands of aggregated AuNPs separated by areas where no graphene decoration was present.

We report here a similar approach for the preparation of RGO-AuNPs nanohybrids, which we have previously employed for the preparation of analogous species with single walled carbon nanotubes (SWCNTs). ${ }^{[32]}$ The strategy involves the synthesis of AuNPs capped with a mixed monolayer of 
choline and pyrene derivatives (PyAuNPs), being the latter useful for the interaction with the carbon nanostructure surface. In the case of SWCNTs, we demonstrated that, by employing $2 \mathrm{~nm}$ PyAuNPs, water soluble nanohybrids were generated, thus being able to make use of the water affinity of choline moieties to extract hydrophobic pristine nanotubes in a polar environment. ${ }^{[32]}$ In the present case we obtain insoluble RGO-AuNPs nanohybrids, characterized by a very homogeneous distribution of AuNPs on the surface of RGO, stating the validity of our mild non-covalent method to obtain welldefined composite materials.

\section{Experimental section}

General: Solvents were purified by standard methods. All commercially reagents were purchased from Sigma-Aldrich, unless not specified, and used as received. Thin layer chromatography analyses were performed using Merck $60 \mathrm{~F}_{254}$ precoated silica gel glass plates. Column chromatography was carried out on Macherey-Nagel silica gel 60 (70-230 mesh). NMR spectra were recorded using a Bruker AV III 500 spectrometer operating at $500 \mathrm{MHz}$ for ${ }^{1} \mathrm{H}, 125.8 \mathrm{MHz}$ for ${ }^{13} \mathrm{C}$. Chemical shifts are reported relative to internal $\mathrm{Me}_{4} \mathrm{Si}$. Multiplicity is given as follow: $\mathrm{s}=$ singlet, $\mathrm{d}=$ doublet, $\mathrm{t}=$ triplet, $\mathrm{q}=$ quartet, $\mathrm{qn}=$ quintet, $\mathrm{m}=$ multiplet, $\mathrm{br}=$ broad peak. HRMS mass spectra were obtained with a Mariner Applied Biosystem (API-TOF) mass spectrometer (MeOH, 0.5\% formic acid). TEM images were recorded on a Jeol 300 PX electron microscope. One drop of sample was placed on the sample grid and the solvent was allowed to evaporate. UV-Visible spectra were recorded on a Cary 50 spectrophotometer equipped with thermostated multiple cell holders. The Raman spectra were acquired with an Xplora spectrometer from Horiba with an excitation line at $632 \mathrm{~nm}$. Excitation photoluminescence spectroscopy was performed with a Nanolog spectrofluorometer from Horiba, equipped with a Xenon lamp and a liquid-nitrogen cooled InGaAs detector.

\subsection{Synthesis of thiol 1 and 2}

Thiol 1 and 2 were prepared according to previously reported procedure. ${ }^{[32,33]}$

\subsection{Synthesis and characterization of monolayer protected gold nanoparticles (T1-NP-a, b)}

NP-a (2 nm) monolayer protected gold nanoparticles were prepared modifying a previously reported two-step procedure. ${ }^{[34]}$ A solution of $\mathrm{HAuCl}_{4} \cdot 3 \mathrm{H}_{2} \mathrm{O}$ (50 mg, $0.127 \mathrm{mmol}, 1$ equiv) in water (2 mL) was extracted with a solution of tetraoctylammonium bromide ( $0.175 \mathrm{~g}, 0.318 \mathrm{mmol}, 2.5$ equiv) in $\mathrm{N}_{2}$ purged toluene $(125 \mathrm{~mL})$. To the resulting reddish-orange organic solution dioctylamine (0.613 g, 2.539 mmol, 20 equiv) was added (the amount of dioctylamine was calculated in order to obtain 2 $\mathrm{nm}$ nanoparticles). The mixture was vigorously stirred under $\mathrm{N}_{2}$ for 1,5 hours. During this period of time the color of the mixture faded. Then a $\mathrm{NaBH}_{4}$ solution (48.0 mg, 1.269 mmol, 10 equiv) in $\mathrm{H}_{2} \mathrm{O}$ $(1 \mathrm{~mL})$ was then rapidly added. The color of the solution turned rapidly to black and after 1.5 hours of stirring at $0^{\circ} \mathrm{C}$, the aqueous layer was removed. To the obtained nanoparticle solution, thiol 2 
(0.254 mmol, 2 equiv) dissolved in $3 \mathrm{~mL}$ of $\mathrm{MeOH}$ was rapidly added. The reaction mixture was stirred overnight. Then the mixture was centrifuged and the precipitated NPs were dissolved in the minimum amount of $\mathrm{MeOH}$, sonicated and then precipitated with EtOAc (5 times); the resulting NPs were finally purified by gel permeation chromatography with Sephadex LH-20.

NP-b (8 nm) monolayer protected gold nanoparticles were prepared modifying a reported procedure. ${ }^{[35]}$ Typically, to $5.6 \mathrm{ml}$ of water, sequentially and under vigorous stirring, $1.6 \mathrm{ml}$ of sodium citrate (510 mM), $250 \mu \mathrm{l}$ of silver nitrate $(10 \mathrm{mM})$ and $500 \mu \mathrm{l}$ of tetrachloroauric acid $(250 \mathrm{mM})$ were added and stirred for 5 minutes. During this time, the solution changed from initial yellow color to green, grey and finally black. After the incubation time, the solution was quickly added to $117 \mathrm{ml}$ of boiling water and heated under reflux for 1 hour, becoming wine-red after a few seconds. The citratecapped nanoparticles solution obtained was then allowed to cool down to room temperature, then thiol 2 was added in 1-2 ml of isopropanol and left stirring overnight. Nanoparticles were purified by using $15 \mathrm{ml}$ Amicon ${ }^{\circledR}$ Ultra filters of $100 \mathrm{kDa}$ molecular weight cutoff centrifuged for 2.5 minutes at $2000 \mathrm{rpm}$ or $10 \mathrm{kDa}$ at $5000 \mathrm{rpm}$ for 6 minutes. Prior to use, filters were prewashed 5 times with 1:1 $\mathrm{H}_{2} \mathrm{O}: \mathrm{MeOH}$, then nanoparticles washed 5 times with 1:1 $\mathrm{H}_{2} \mathrm{O}: \mathrm{MeOH}$ and 5 times with pure water. Typical concentrated volumes after centrifugation for 15, 4 and $0.5 \mathrm{ml}$ filtering units were 1.5, 0.5 and $0.1 \mathrm{ml}$ respectively. Purified nanoparticles aqueous solution were frozen with dry ice and freezedried overnight on a 5Pascal 105PDGT lyophilizer equipped with and Edwards XDS 10 Pump (P $\approx$ 0.2 mbar, $\mathrm{T}=-50^{\circ} \mathrm{C}$ ). Typically, an amount between 22 and $26 \mathrm{mg}$ of functionalized nanoparticles was obtained, corresponding to $c a$. $75 \%$ yield.

Nanoparticles were analyzed by NMR in situ by detaching the thiols from its surface by treating 500 $\mu \mathrm{l}$ of nanoparticles solution with $100 \mu \mathrm{l}$ of a saturated solution of iodine in deuterated solvent.

\subsubsection{Thiol exchange reaction}

T1-NP-a: In a sealed vial, under $\mathrm{N}_{2}$ atmosphere, 0.5 equiv of thiol $\mathbf{1}$ were added to a MeOH solution (3 mL) of T2-NP-a (20 mg) and the solution was reacted at room temperature for 16 hours. The resulting NPs T1-NP-a were finally purified by gel permeation chromatography using Sephadex LH20 resin.

T1-NP-b: In a sealed vial, under $\mathrm{N}_{2}$ atmosphere, 20 equiv of thiol 1 were added to a MeOH solution (2 mL) of T2-NP-b (10 mg) and the solution was reacted at room temperature for 16 hours.

The resulting NPs T1-NP-b were finally purified by using Amicon ${ }^{\circledR}$ Ultra filters of $100 \mathrm{KDa}$ molecular weight cutoff.

\subsubsection{Characterization of T1-NP-a.}

TEM analysis of the different samples of small nanoparticles T1-NP-a (Fig. 1) yields an average diameter of $2.1 \pm 0.2 \mathrm{~nm}$. NMR analysis (Fig. 2) indicates monolayer formation (broadening of all 
signals), as confirmed by diffusion-filtered experiments (not shown). Composition of T1-NP-a was determined to be $30 \%$ after integration of distinctive 1 NMR signals.
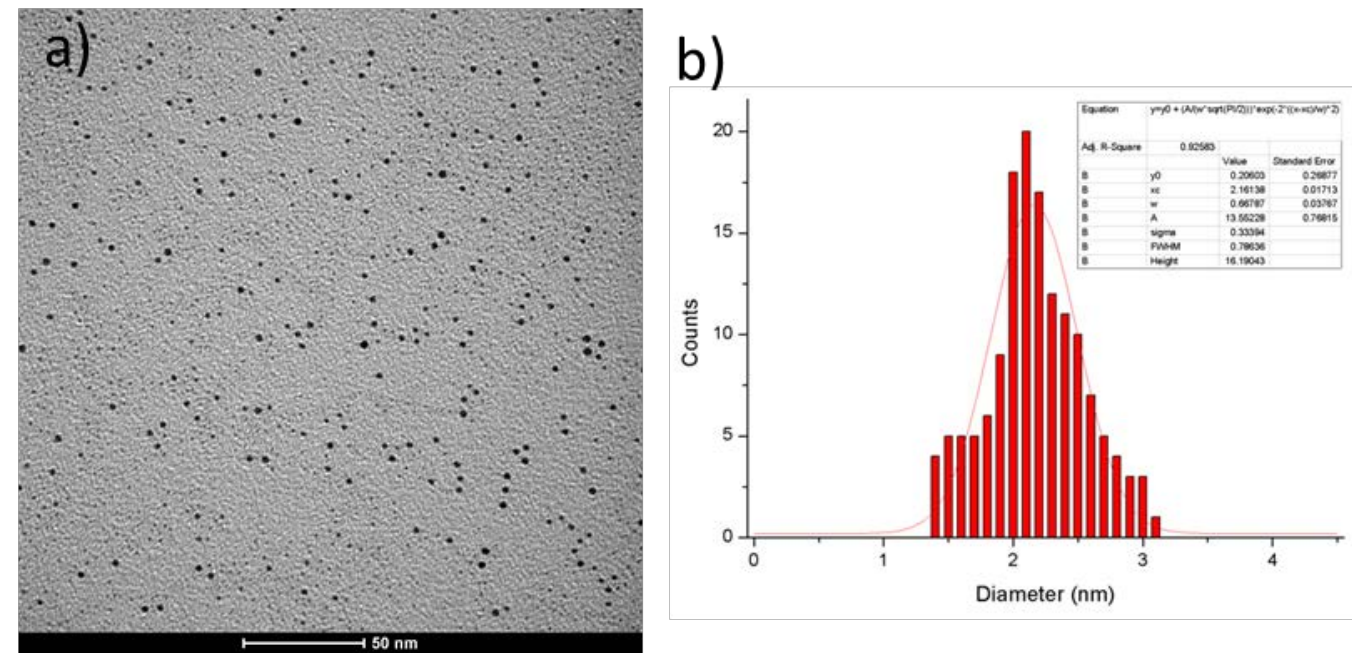

Figure 1. a) Low resolution TEM image of T1-NP-a and b) size distribution (average diameter $=2.1 \pm 0.2 \mathrm{~nm}$ ).

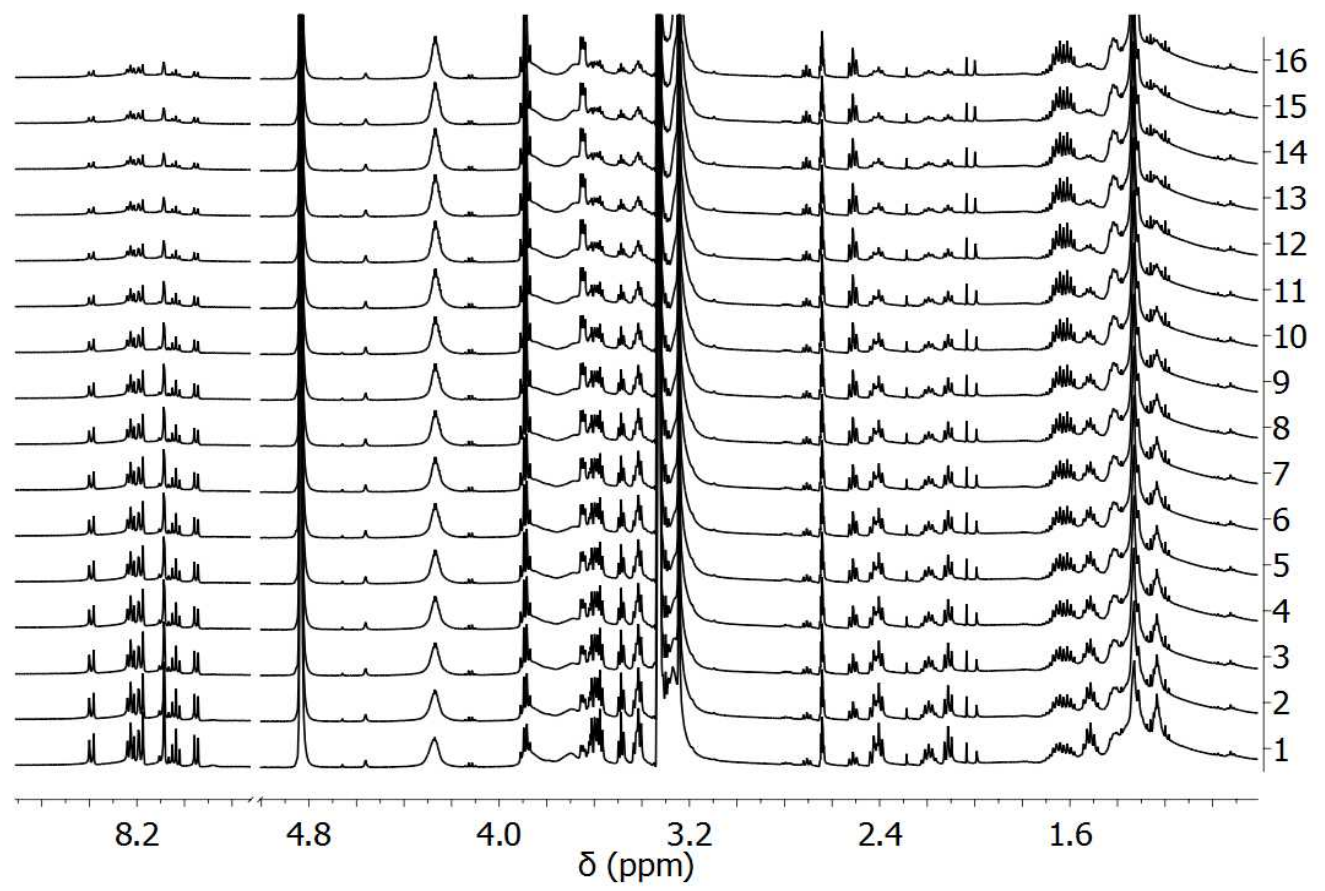

Figure 2. ${ }^{1} \mathrm{H}$ NMR spectrum of T1-NP-a thiol exchange reaction. Conditions: $1.2 \mathrm{mg}$ of thiol 1, $3.8 \mathrm{mg}$ of T2-NP-a in $0.6 \mathrm{ml}$ of MeOD. Spectrum 1 was acquired immediately after the addition of thiol 1; subsequent $1 \mathrm{H}-\mathrm{NMR}$ spectra were acquired every hour.

\subsubsection{Characterization of T1-NP-b.}

TEM analysis of the different samples of T1-NP-b (Fig. 3) yields an average diameter of $8.2 \pm 0.5 \mathrm{~nm}$. NMR analysis (Fig. 4) indicates monolayer formation (broadening of all signals). Composition of T1NP-a was determined to be 30\% after integration of distinctive 2 NMR signals. 

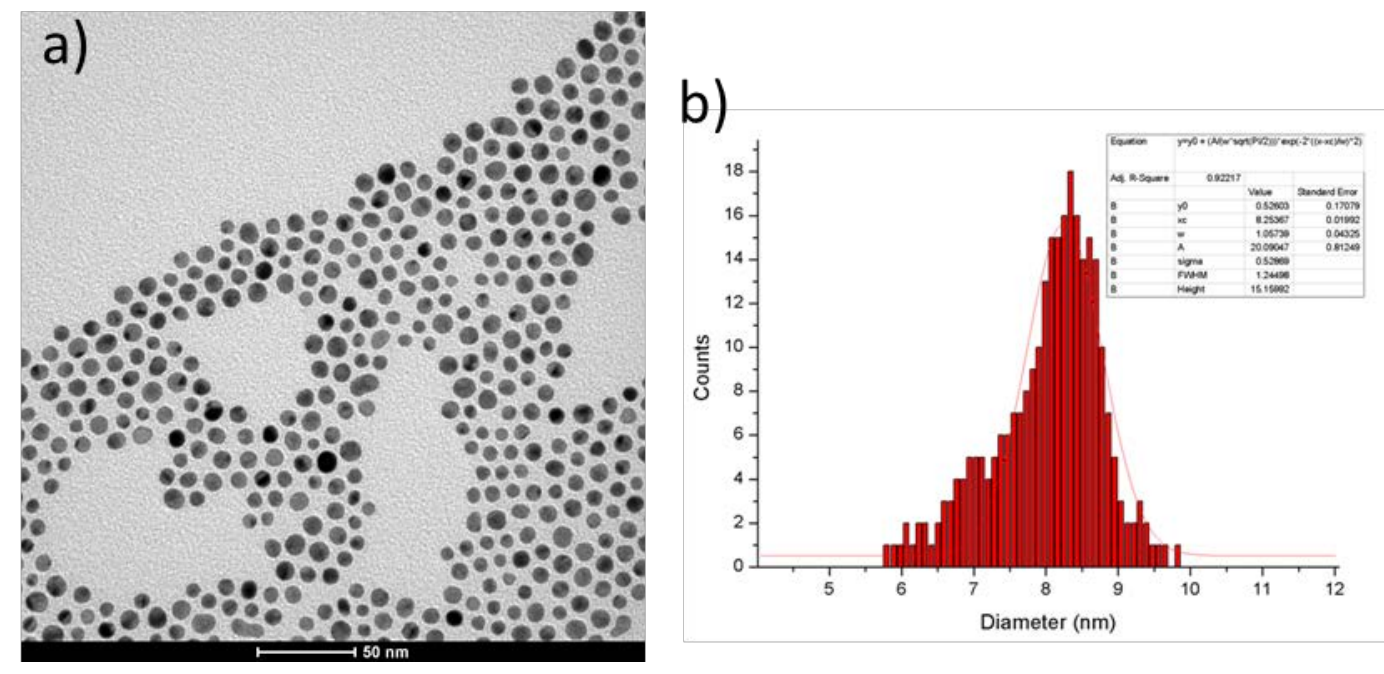

Figure 3. a) Low resolution TEM image of T1-NP-b and b) size distribution (average diameter $8.2 \pm 0.5 \mathrm{~nm}$ ).

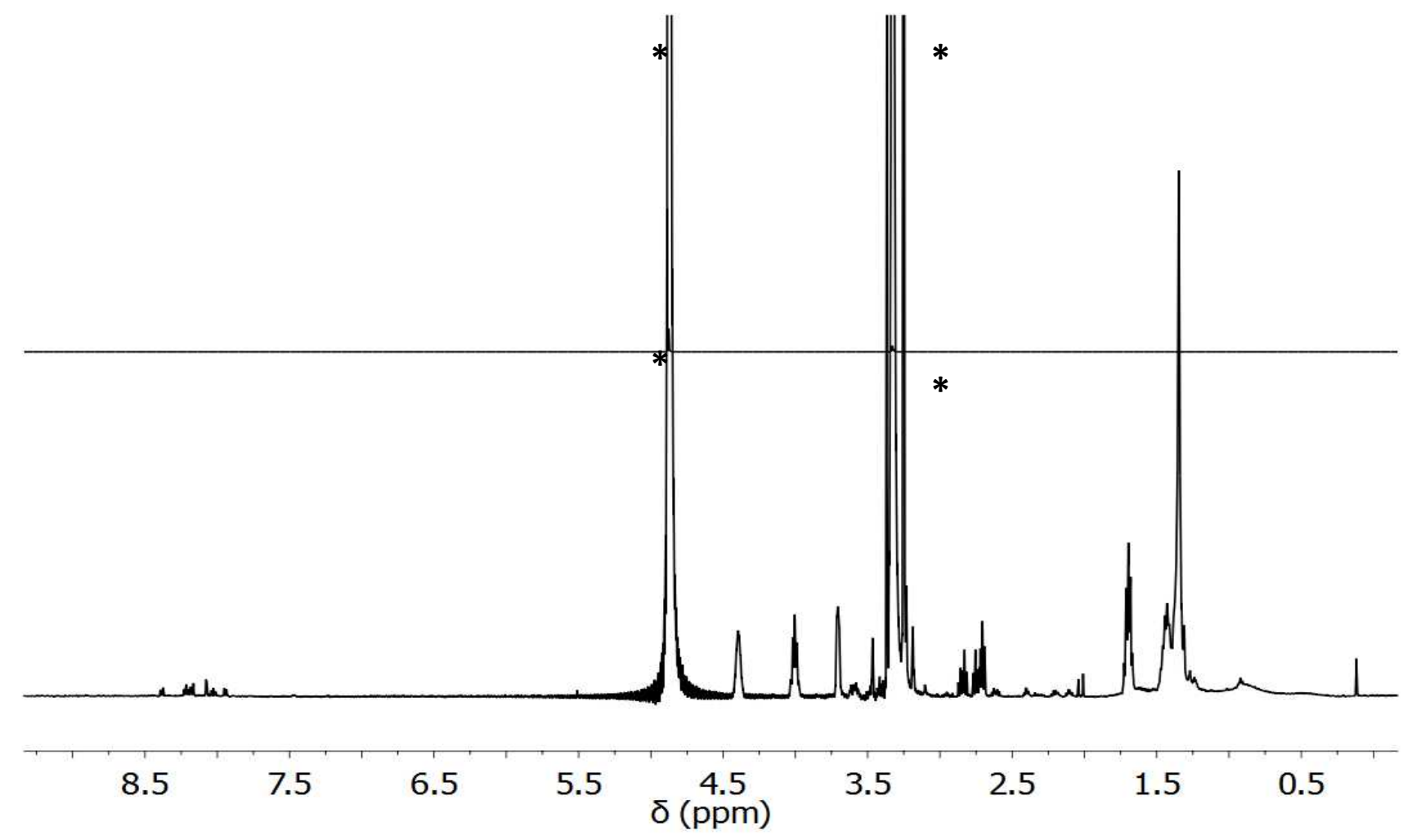

Figure 4. ${ }^{1} \mathrm{H}$ NMR spectrum of T1-NP-b in MeOD before (Top) and after (Bottom) $\mathrm{I}_{2}$ oxidation ( indicates the residual solvents and impurities). No signals are visible in the NP spectrum before oxidation since the slow nanoparticles tumbling rate causes signal broadening beyond detectability.

\subsection{Decoration of RGO with PyAuNPs}

$2 \mathrm{mg}$ of T1-NP-a, b were dissolved in $5 \mathrm{~mL}$ of milliQ $\mathrm{H}_{2} \mathrm{O}$, then $1 \mathrm{mg}$ of RGO (purchased from ACS Material, LLC, product No.: GnP1L-0.5g, and used as received) was added and the suspension was sonicated for 1 hour $\left(1.2 \mathrm{~W} \mathrm{~mL}^{-1}\right)$. The resulting solutions turned clear, indicating that the majority of the AuNPs were bound to the graphene and that the formed hybrid material was insoluble in water. The obtained precipitate was purified by washing it in water for 5 times. 


\subsection{Preparation of the SWCNTs-PyAuNPs and SWCNTs-Py hybrids}

CoMoCATs nanotubes by SouthWest NanoTechnologies (SWeNT, SG 76, diameter of $(0.9 \pm 0.3) \mathrm{nm}$ and high aspect ratio (>1000)), were dispersed in water at a concentration of $0.1 \mathrm{~g} \mathrm{~L}^{-1} .4 \mathrm{~mL}$ of the water-SWCNTs mixture were then treated with additional $0.5 \mathrm{~mL}$ of, respectively: a) T1-NP-a for the SWCNTs-PyAuNPs sample (T1-NP-a concentration $=0.4 \mathrm{mg} \mathrm{mL}^{-1}$; pyrene concentration in T1$\mathrm{NP}-\mathrm{a}=30 \mathrm{wt} \%)$ and b) pyrene-derivative for the SWCNTs-Py sample $\left(3.5 \times 10^{-5} \mathrm{M}\right)$. The concentrations of the pyrene-derivative and of T1-NP-a solutions were adjusted to ensure the same amount of pyrene in both solutions. To further promote exfoliation of the nanotube bundles and stability of the final suspensions, sodium cholate was further added to the solutions (1 wt\%). The two solutions were then sonicated with a tip-sonicator (Bandelin SonoPlus HD 2070) at a power of $60 \mathrm{~W}$ and cycles of $0.5 \mathrm{~s}$. After 1h, the suspensions were centrifuged (Hettig Mikro 220R centrifuge) at $3500 \mathrm{rpm}$ at $27^{\circ} \mathrm{C}$ for $60 \mathrm{~min}$. The collected supernatant was used for the experiments.

\section{Results and discussion}

Differently from our previous work with SWCNTs, ${ }^{[32]}$ this time we choose to test the same $2 \mathrm{~nm}$ PyAuNPs and another set of PyAuNPs with a larger diameter $(8 \mathrm{~nm})$, thus featuring a plasmonic contribution in the visible range (see the absorption spectrum in Fig. 7). The synthesis of the mixed monolayer particles capped with choline and pyrene derivatives (Fig. 5) was performed according to published procedures. ${ }^{[32,33]}$
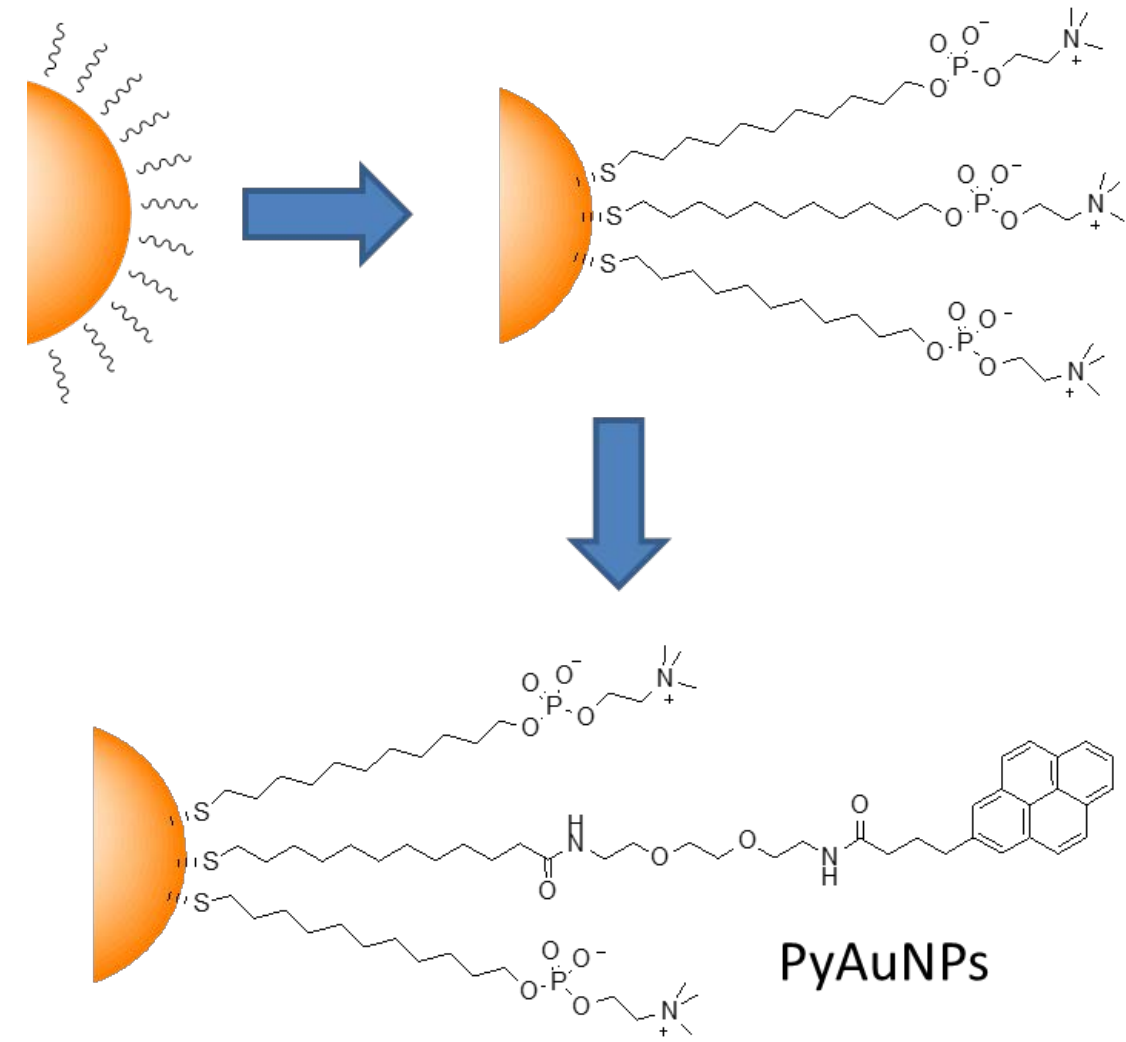

Figure 5. Graphical sketch of the synthesis of PyAuNPs. 
Smaller nanoparticles were prepared by reduction of tetrachloroaurate in toluene with $\mathrm{NaBH}_{4}$ in the presence of dioctylamine, while larger ones were prepared by silver seeded reduction of tetracholoroaurate in water with sodium citrate. In our previous work, ${ }^{[32]}$ we showed that direct formation of the mixed monolayers made by thiols 1 and 2 through simultaneous addition of the two coating molecules did not allow to obtain nanoparticles with 1 content higher than $15 \%$. For this reason, we decided to prepare the mixed monolayers by thiols exchange. 2-coated AuNPs were prepared by exchanging the weak stabilizing agents (dioctylamine and citrate respectively in the case of 2 and 8 nm diameter nanoparticles) and then subjected to exchange with thiol 1 in methanol (T1AuNP-a). After equilibration (followed by NMR, see Fig. 2) the nanoparticles were purified by size exclusion chromatography and the monolayer composition was determined by ${ }^{1} \mathrm{H}-\mathrm{NMR}$ after thiol detachment by $\mathrm{I}_{2}$ oxidation.

We found that monolayer coating 2 nm PyAuNPs contained 30\% of 1 thiols, while the one coating 8 nm PyAuNp contained 20\%. Interestingly, substitution required much larger amounts (20 equivalents) of entering thiols with large nanoparticles than with the small ones (0.5 equivalents), suggesting that the higher curvature favors the mixed monolayer either for kinetic or thermodynamic reasons. Both the nanoparticles are well soluble in water and methanol, and NOE-NMR experiments (not shown) suggest that the pyrene groups are, as expected, bent into the monolayer formed by the alkyl chains of the thiols.

The initial idea in the preparation of the two types nanohybrids of PyAuNPs with RGO was that of obtaining, as it was for the case of the nanoybrids with SWCNTs, ${ }^{[32]}$ water solubilization of the GBM by means of the AuNPs bearing zwitterionic pendant choline groups. In this way, multivalency ${ }^{[31]}$ on the AuNPs would have been exploited, allowing these last ones to act similarly to surfactants for RGO. Differently from common surfactants anyway, PyAuNPs are expected to be selective for individual sheets rather than for small RGO aggregates, which are sometimes found within standard surfactant micelles dispersing carbon nanomaterials in water. Unfortunately things went differently, being RGO the species driving the solubility profile of the final hybrid. The procedure (described in the Experimental Section) involving addition of pristine RGO to a water solution of the PyAuNPs followed by sonication and ultracentrifugation to eliminate major aggregates, lead to the total precipitation of all the material present in solution, with no residual free particle in the supernatant. This indicates that the affinity of the pyrene functional groups on the AuNPs for RGO is very high and that all the particles are more keen to attach on this last one than to remain in water solution. It is likely that an upper limit to the density of PyAuNPs attached to the RGO surface is given by the lack of residual space on the conjugated carbon based nano-platform. This anyway remains subject of future studies. By looking at the TEM images of the precipitated materials (Fig. 6), it is evident a very 
high degree of coverage of the RGO surface, characterized by a pronounced homogeneity, particularly fo $r$ the case of the PyAuNPs-RGO hybrid with the smaller particles (Fig. 6a). In the hybrid with the bigger particle, a higher amount of AuNPs aggregates onto the RGO surface is present (Fig. 6 b).

The formation of aggregates of $8 \mathrm{~nm}$ PyAuNPs in the hybrid with RGO is also somehow evident from the UV-visible-NIR spectrum of the hybrid, which features a very weak shoulder over the continuous absorption contribution of RGO in water (highly scattering and thus completely flat from UV to NIR) that is red-shifted (very noisy signal with a maximum at around $604 \mathrm{~nm}$ ) with respect to the plasmonic band of the free $8 \mathrm{~nm}$ PyAuNPs (maximum at $541 \mathrm{~nm}$ ) and thus can be attributed to aggregated particles (Fig. 7a). ${ }^{[36]}$

a)

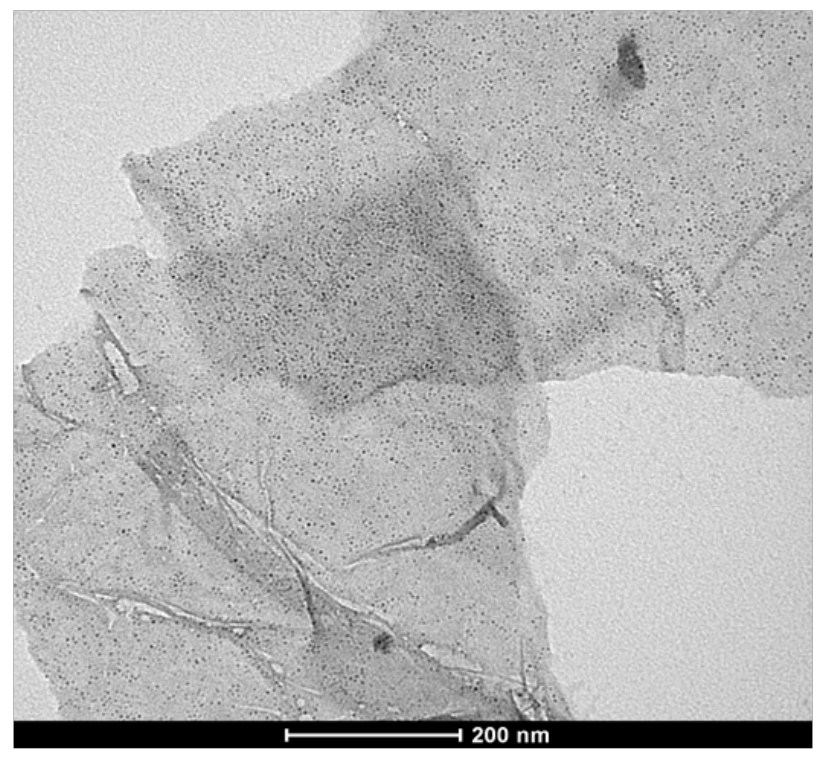

b)

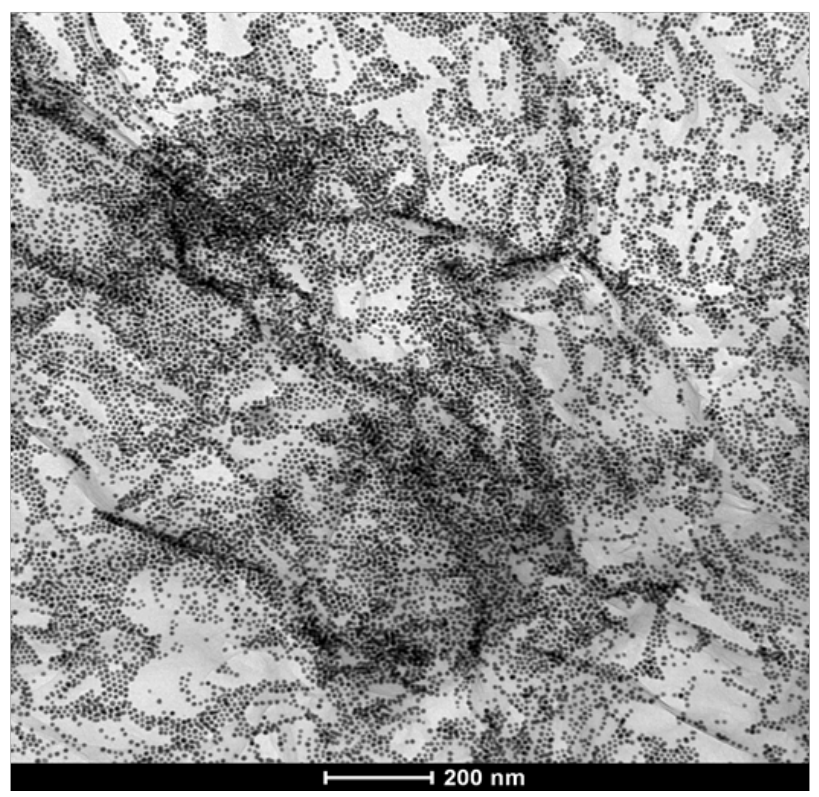

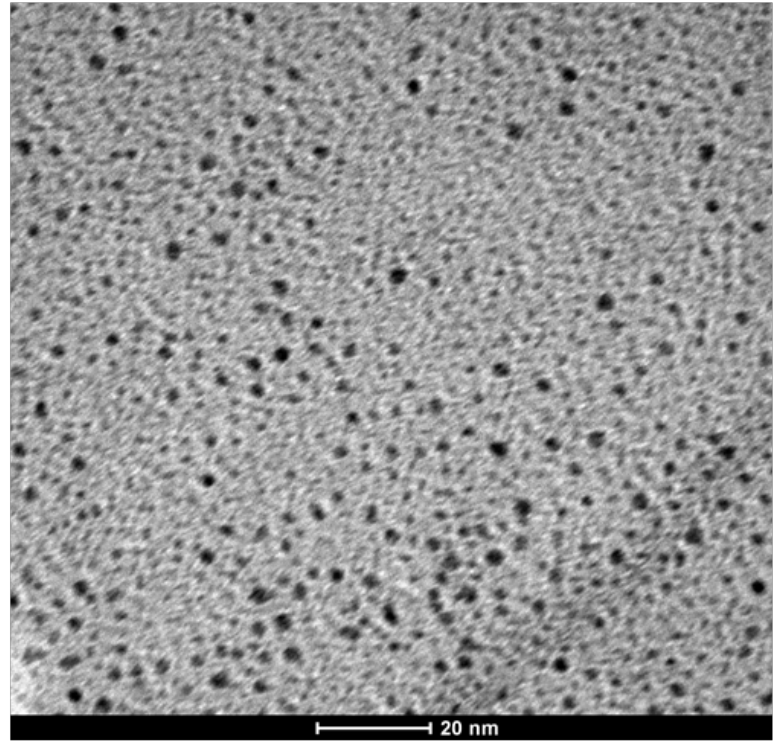

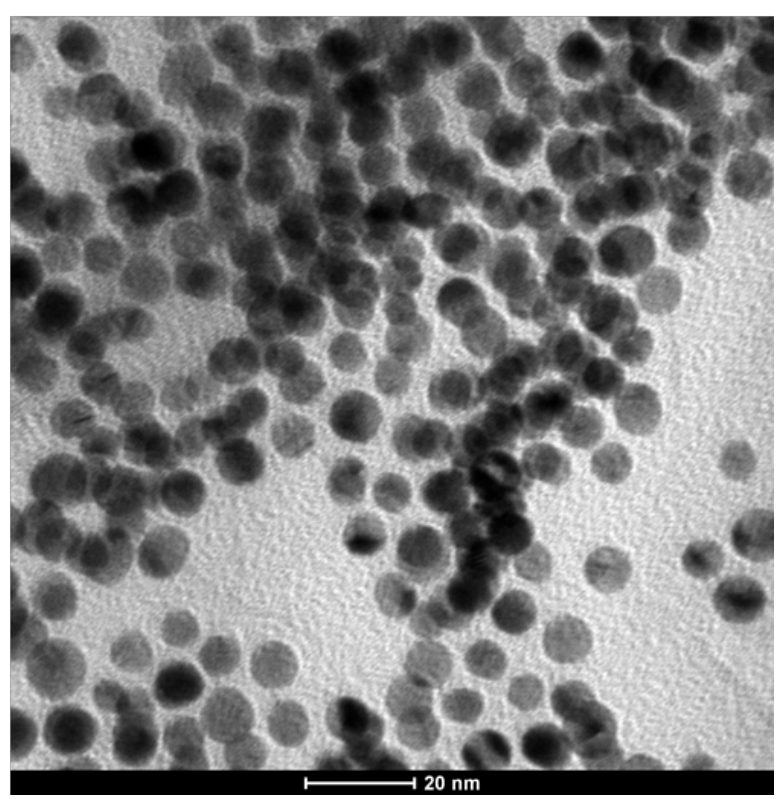

Figure 6. TEM images of PyAuNPs-RGO nanohybrids with a) 2 nm PyAuNPs and b) 8 nm PyAuNPs. 
To probe the nature of interaction between PyAuNPs and the RGO surface, the RGO-PyAuNPs composites were analyzed for their Raman spectra. We recorded the spectra of RGO-PyAuNPs (2 $\mathrm{nm}$ ) composite and compared it to the one of bare RGO (we were not able to get a spectrum from RGO-PyAuNPs -8 nm- composite due to due to the increased scattering losses by the bigger particle). The comparison between the two spectra is reported in Fig. 7 b. The typical D and G bands of a GBM are found in the Raman spectrum of the RGO-PyAuNPs at 1336 and $1586 \mathrm{~cm}^{-1}$, respectively. The two main bands for the RGO derivative resemble those present in the Raman spectrum of pristine RGO, but with different $\mathrm{I}_{\mathrm{D}} / \mathrm{I}_{\mathrm{G}}$ ratio. This last parameter appears to be slightly higher in the composite, indicating at first approximation the generation of further defects on the RGO surface ${ }^{[37]}$ following non-covalent functionalization with PyAuNPs. More properly, the reason for higher $\mathrm{I}_{\mathrm{D}} / \mathrm{I}_{\mathrm{G}}$ ratio in the hybrid with respect to the pristine GBM might be related to the reduction in size of single aromatic clusters present on RGO, due to the presence of the particles that act like local interrupters of aromaticity. ${ }^{[37]}$ Notwithstanding that, what results to be more striking from the two spectra is the enhancement in the Raman signal of RGO when coupled to AuNPs. The signal of RGO in the hybrid is indeed found to be enhanced by $\sim 3$ times in the presence of the AuNPs. This observed Raman signal enhancement is similar to what found by Jasuja et al. for composites of AuNPs and graphene oxide $^{[38]}$ and paves the way to the application of our hybrid systems as functional platforms for surface enhanced Raman scattering (SERS) detection of analytes. ${ }^{[39]}$

As no direct band-band recombinative emission can be observed from RGO, we tested other semiconducting carbon-based nanosystems to further support the evidence of PyAuNPs-promoted optical response enhancement.

a)

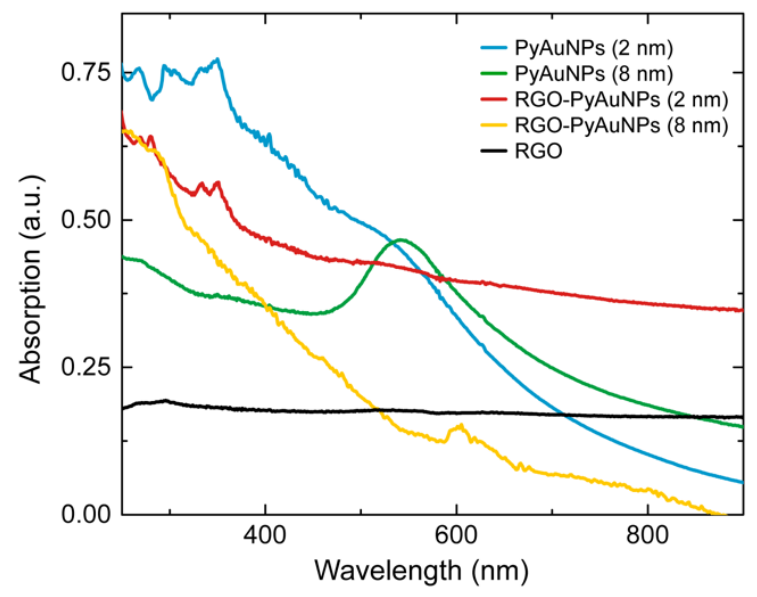

b)

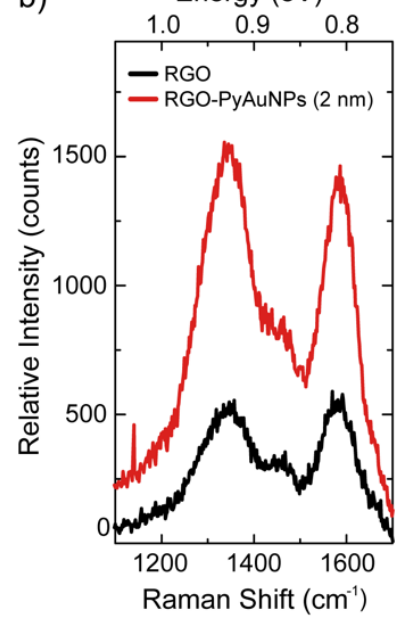

c)

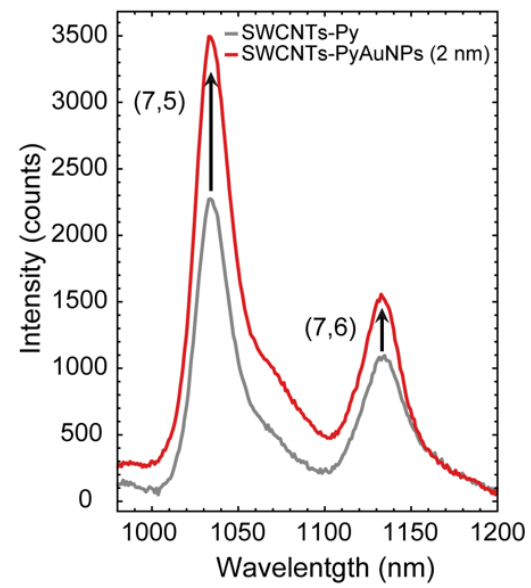

Figure 7. a) UV-visible-NIR absorption spectra of PyAuNPs (2 and $8 \mathrm{~nm}$ ) and their composites with RGO (the absorption of bare RGO is also reported) in water dispersion. b) Raman spectra of RGO compared with RGO-PyAuNPs collected from powder samples. c) Emission spectra of the $(7,5)$ and $(7,6)$ SWCNT species excited at $650 \mathrm{~nm}$ in the presence of the Py derivative and of PyAuNPs (2 nm). 
To this purpose, we realized a novel set of nanohybrids incorporating SWCNTs and analyzed them through photoluminescence (PL) spectroscopy. Close proximity between gold nanoparticles and SWCNTs has already been demonstrated to enhance the SWCNT emission yield. ${ }^{[40,41]}$ We accordingly prepared two set of samples, namely SWCNTs coated just with the pyrene-derivative (SWCNTs-Py) and SWCNTs coated with PyAuNPs (2 nm; SWCNTs-PyAuNPs). The samples have been produced by ensuring that the same amount of SWCNTs was present in both, so that the emission intensities are directly comparable and related to the emission yields. Fig. 7c compares the emission spectra collected by the two set of samples, clearly showing the gold-assisted enhancement of the emission from the hybrid carbon-gold system, interacting by means of the Py functionalities present on the AuNPs.

\section{Conclusions}

We have presented here and characterized a new series of RGO-AuNP hybrids obtained through a non-covalent strategy, in which the assembly of the pristine species proceeds mildly in water at room temperature. The approach is based on exploiting the versatility of AuNPs for being decorated with different functional groups, introduced on the surface resorting to Au-S covalent bonds.

The selected AuNPs contain indeed a mix monolayer in which pendant pyrene functionalities are present (PyAuNPs), which are able to establish with the surface of conjugated carbon nanostructures high-affinity interactions through $\pi$ - $\pi$ stacking. In this way, a very homogenous and high coverage of the RGO surface with the AuNPs is achieved, generating well-defined entities which demonstrate to have the full potential for a use as platforms for SERS analysis. This remains therefore the subject of our future investigations.

\section{Acknowledgements}

LG, GA and FM acknowledge financial support from the European Union with the ERC Starting grant "MOSAIC" (n. 259014) and the University of Padova with the Progetto Strategico di Ateneo "NAMECA". EM and TG acknowledge financial support from the University of Padova with the contract P-DiSC \#05BIRD2016-UNIPD. MG, SJ, SR, and AS gratefully acknowledge financial support from the German Research Foundation (DFG via SFB 658, subproject A6) and from the Focus Area NanoScale of the Freie Universität Berlin.

\section{References}

[1] P. Wick, A. E. Louw-Gaume, M. Kucki, H. F. Krug, K. Kostarelos, B. Fadeel, K. A. Dawson, A. Salvati, E. Vázquez, L. Ballerini, M. Tretiach, F. Benfenati, E. Flahaut, L. Gauthier, M. Prato, A. Bianco, Angew. Chem. Int. Ed. 2014, 53, 7714.

[2] Y.-C. Yeh, B. Creran, V. M. Rotello, Nanoscale 2012, 4, 1871.

[3] D. Su, Y. Zhang, Z. Wang, Q. Wan, N. Yang, Carbon 2017, 117, 313.

[4] X. Qiu, W. Zhou, C. Qi, D. Cheng, Y. Ouchi, ChemElectroChem 2017, n/a.

[5] Y. Zhao, Y. Zhu, Nanoscale 2015, 7, 14561.

[6] A. Rani, D. B. Velusamy, F. Marques Mota, Y. H. Jang, R. H. Kim, C. Park, D. H. Kim, Adv. Funct. Mater. 2017, 27, 1604604. 
[7] G. Sahoo, N. Sarkar, D. Sahu, S. K. Swain, RSC Adv. 2017, 7, 2137.

[8] P. T. Yin, S. Shah, M. Chhowalla, K.-B. Lee, Chem. Rev. 2015, 115, 2483.

[9] D. Rodrigo, O. Limaj, D. Janner, D. Etezadi, F. J. García de Abajo, V. Pruneri, H. Altug, Science 2015, 349,

[10] G. Zhao, H. Wang, G. Liu, Z. Wang, J. Cheng, Ionics 2017, 23, 767.

[11] Y. Wang, Y. Zhang, D. Wu, H. Ma, X. Pang, D. Fan, Q. Wei, B. Du, Sci. Rep. 2017, 7, 42361.

[12] Y. Zhao, D. Yang, X. Li, Y. Liu, X. Hu, D. Zhou, Y. Lu, Nanoscale 2017, 9, 1087.

[13] G. Modugno, C. Ménard-Moyon, M. Prato, A. Bianco, Br. J. Pharmacol. 2015, 172, 975.

[14] K. S. Novoselov, A. K. Geim, S. V. Morozov, D. Jiang, Y. Zhang, S. V. Dubonos, I. V. Grigorieva, A. A. Firsov, Science 2004, 306, 666.

[15] A. A. Balandin, S. Ghosh, W. Bao, I. Calizo, D. Teweldebrhan, F. Miao, C. N. Lau, Nano Lett. $2008,8,902$.

[16] C. Lee, X. Wei, J. W. Kysar, J. Hone, Science 2008, 321, 385.

[17] R. R. Nair, P. Blake, A. N. Grigorenko, K. S. Novoselov, T. J. Booth, T. Stauber, N. M. R. Peres, A. K. Geim, Science 2008, 320, 1308.

[18] E. V. Canesi, M. Capsoni, L. Karnam, A. Lucotti, C. Bertarelli, M. Del Zoppo, J. Phys. Chem. C 2013, 117, 13197.

[19] M.-C. Daniel, D. Astruc, Chem. Rev. 2004, 104, 293.

[20] E. Katz, I. Willner, Angew. Chem. Int. Ed. 2004, 43, 6042.

[21] B. Perrone, S. Springhetti, F. Ramadori, F. Rastrelli, F. Mancin, J. Am. Chem. Soc. 2013, $135,11768$.

[22] Y. Zhang, L. Zhang, C. Zhou, Acc. Chem. Res. 2013, 46, 2329.

[23] Y. Li, N. Chopra, JOM 2015, 67, 34.

[24] S. Abdolhosseinzadeh, H. Asgharzadeh, H. Seop Kim, Sci. Rep. 2015, 5, 10160.

[25] S. Zoladek, I. A. Rutkowska, M. Blicharska, K. Miecznikowski, W. Ozimek, J. Orlowska, E. Negro, V. Di Noto, P. J. Kulesza, Electrochim. Acta 2017, 233, 113.

[26] J. Liu, X. Xin, H. Zhou, S. Zhang, Chem. Eur. J. 2015, 21, 1908.

[27] A. T. E. Vilian, S.-K. Hwang, C. H. Kwak, S. Y. Oh, C.-Y. Kim, G.-w. Lee, J. B. Lee, Y. S. Huh, Y.-K. Han, Synth. Met. 2016, 219, 52.

[28] J. Park, H. S. N. Jayawardena, X. Chen, K. W. Jayawardana, M. Sundhoro, E. Ada, M. Yan, Chem. Commun. 2015, 51, 2882.

[29] H. Ismaili, D. Geng, A. X. Sun, T. T. Kantzas, M. S. Workentin, Langmuir 2011, 27, 13261.

[30] E. M. Perez, N. Martin, Chem. Soc. Rev. 2015, 44, 6425.

[31] F. G. Brunetti, H. Isla, J. Aragó, E. Ortí, E. M. Pérez, N. Martín, Chem. Eur. J. 2013, 19, 9843.

[32] P. Salice, A. Gambarin, N. Daldosso, F. Mancin, E. Menna, J. Phys. Chem. C 2014, 118, 27028.

[33] R. E. Holmlin, X. Chen, R. G. Chapman, S. Takayama, G. M. Whitesides, Langmuir 2001, $17,2841$.

[34] F. Manea, C. Bindoli, S. Polizzi, L. Lay, P. Scrimin, Langmuir 2008, 24, 4120.

[35] H. Xia, S. Bai, J. Hartmann, D. Wang, Langmuir 2010, 26, 3585.

[36] R. Elghanian, J. J. Storhoff, R. C. Mucic, R. L. Letsinger, C. A. Mirkin, Science 1997, $277,1078$.

[37] A. C. Ferrari, J. Robertson, Phys. Rev. B 2000, 61, 14095.

[38] K. Jasuja, J. Linn, S. Melton, V. Berry, J. Phys. Chem. Lett. 2010, 1, 1853.

[39] G. Lu, H. Li, C. Liusman, Z. Yin, S. Wu, H. Zhang, Chem. Sci. 2011, 2, 1817.

[40] M. Glaeske, A. Setaro, Nano Res. 2013, 6, 593.

[41] A. Setaro, M. Adeli, M. Glaeske, D. Przyrembel, T. Bisswanger, G. Gordeev, F. Maschietto, A. Faghani, B. Paulus, M. Weinelt, R. Arenal, R. Haag, S. Reich, Nat. Commun. 2017, 8, 14281. 